\title{
Feedback Prediction for Proactive HARQ in the Context of Industrial Internet of Things
}

\author{
Barış Göktepe*, Tatiana Rykova*, Thomas Fehrenbach*, Thomas Schierl* and Cornelius Hellge* \\ *Video Coding \& Analytics Department \\ Fraunhofer Heinrich Hertz Institute, Berlin, Germany \\ Email: first.last@hhi.fraunhofer.de
}

\begin{abstract}
In this work, we investigate proactive Hybrid Automatic Repeat reQuest (HARQ) using link-level simulations for multiple packet sizes, modulation orders, BLock Error Rate (BLER) targets and two delay budgets of $1 \mathrm{~ms}$ and $2 \mathrm{~ms}$, in the context of Industrial Internet of Things (IIOT) applications. In particular, we propose an enhanced proactive HARQ protocol using a feedback prediction mechanism. We show that the enhanced protocol achieves a significant gain over the classical proactive HARQ in terms of energy efficiency for almost all evaluated BLER targets at least for sufficiently large feedback delays. Furthermore, we demonstrate that the proposed protocol clearly outperforms the classical proactive HARQ in all scenarios when taking a processing delay reduction due to the less complex prediction approach into account, achieving an energy efficiency gain in the range of $11 \%$ up to $15 \%$ for very stringent latency budgets of $1 \mathrm{~ms}$ at $10^{-2}$ BLER and from $4 \%$ up to $7.5 \%$ for less stringent latency budgets of $2 \mathrm{~ms}$ at $10^{-3}$ BLER. Furthermore, we show that power-constrained proactive HARQ with prediction even outperforms unconstrained reactive HARQ for sufficiently large feedback delays.
\end{abstract}

Index Terms-5G mobile communication, Early HARQ, IIOT, Proactive HARQ, Feedback Prediction, Low latency communication, Physical layer, Machine learning, HARQ, Tactile Internet, Machine-type communication

\section{INTRODUCTION}

\section{A. Background and Motivation}

Since the completion of Rel. 15 and Rel. 16 Fifth Generation $(5 \mathrm{G})$ specifications by the 3rd Generation Partnership Project (3GPP), the focus has been shifted from rather generic solutions to cater for the main use cases to more sophisticated solutions targeting the emerging use cases. Tactile Internet (TI) is one of the focus areas of the 3GPP. Especially machine-tomachine type communication, puts demanding requirements on the latency and reliability while keeping the device complexity and power consumption low. Hence, study and work items have been approved to evaluate reduced complexity devices in the context of Industrial Internet of Things (IIOT) services [1], [2]. The frequently mentioned Ultra-Reliable Low Latency Communication (URLLC) use case with a packet error rate smaller than $10^{-5}$ and $1 \mathrm{~ms}$ end-to-end latency [3] is one of the main goals of the further enhancements. However, as previously mentioned, use cases with more stringent latency targets have also turned towards low-complexity and battery powered devices, e.g. safety related sensors [2]. Here, complexity constraints, such as energy or bandwidth limitation, should also be ensured given the demanding URLLC targets. In this work, we focused mainly on the latency and energy constraints since reliability can also be partly moved to higher layer protocols exploiting link diversity, such as Packet Data Convergence Protocol (PDCP) duplication [4].

Lately, Hybrid Automatic Repeat reQuest (HARQ) has been extensively investigated in the context of URLLC in [4], which resulted in a follow-up work item specification. HARQ is a physical layer retransmission mechanism, which enhances spectral efficiency significantly especially in a high reliability regime. However, the major drawback of the so-called reactive HARQ, which is one of the fundamental mechanisms of Long Term Evolution (LTE) and 5G, is an introduced latency due to the HARQ Round Trip Time (RTT). It has to be noted that the reactive Incremental Redundancy (IR) HARQ approaches the ergodic channel capacity of Rayleigh block fading channels [5] and shows a significantly higher energy efficiency compared to other HARQ combination schemes, such as chase-combining [6]. According to this scheme the transmitter splits the codeword, which is generated by the so-called mother code, into subcodewords also designated as Redundancy Versions (RVs) in the 3GPP context. These $\mathrm{RVs}$ are transmitted one by one based on the receiver's feedback, where ACK indicates a successful decode and NACK - an unsuccessful one. However, the HARQ RTT is a major bottleneck of the reactive HARQ [7], and can be reduced by shortening Transmission Time Interval (TTI) to one Orthogonal Frequency Division Multiplexing (OFDM) symbol [7]. Nevertheless, this imposes higher requirements on both the receiver's instantaneous processing bandwidth and the transmitter's power constraint. Furthermore, even performing the transmissions on small multiples of OFDM symbols can reduce the latency only to a certain limit defined by the HARQ RTT. Hence, new HARQ solutions that enable low-latency and reduce the instantaneous bandwidth for low complexity devices are of great importance for investigation.

In Rel. 16 a new HARQ mechanism designated as proactive HARQ was introduced for UpLink (UL) Grant-Free (GF) communication. In compliance with this scheme, the transmitter determines a number of RVs that is required to achieve the reliability target. This number of RVs is transmitted autonomously unless a positive ACK is received before [7], [8]. This scheme solves the HARQ RTT latency issue and provides high reliability at very low latency constraints at the 
expense of spectral efficiency loss [9]. However, it may lead to the transmission of unnecessary RVs due to the feedback delay, which is comprised of the delay components for processing, queuing in the upper layers, propagation delays and feedback transmission time.

Strategies for reducing the feedback delay using prediction mechanisms have been broadly studied in literature. Different HARQ feedback prediction methods based on estimating the channel state are proposed in [10]-[13]. Furthermore, authors in [10] investigate a mixture of proactive and reactive HARQ protocols to reduce the expected latency. In particular, the impact of prediction errors is studied in [11]. In [14], [15], the authors use a Bit Error Rate (BER) estimate based on LogLikelihood Ratios (LLRs) to predict the decoding outcome ahead of the actual decoding. Authors in [16] analyze an approach where an unnecessary decoding of Low-Density Parity-Check (LDPC) codes is avoided in case of predicted packet loss and thus, the feedback delay is reduced. The machine learning techniques that predict the decoding outcome ahead of full reception of the codeword are studied in [17]. In [18] and [19], authors put the prediction methods into the context of cloud-Radio Access Networks (RANs) and show the benefits of early feedback in scenarios with a non-ideal backhaul.

\section{B. Contributions and Organization}

The contributions of this paper are summarized as follows:

- We put forward the feedback prediction approach using a logistic regression on LDPC subcodes [17] proposing an enhanced proactive HARQ protocol, and evaluate its performance on TDL-C fading channels using link-level simulations for different scenarios, i.e. target Block Error Rates (BLERs), feedback delays, Transport Block (TB) sizes, and delay budgets.

- In the second part, we evaluate further benefits of using a feedback prediction in the context of proactive HARQ considering a reduction of the feedback delay compared to the full decoding. We show that reducing the feedback delay by employing feedback prediction provides a significant benefit especially for low BLERs of up to $14 \%$ and up to $8 \%$ for $1 \mathrm{~ms}$ and $2 \mathrm{~ms}$ delay budgets, respectively.

- Furthermore, we show that the proposed proactive HARQ scheme while catering to power constraints, also outperforms reactive HARQ without any power constraint at the same $E_{b} / N_{0}$ ratio for sufficiently high feedback delays relative to the total latency budget, especially in the low BLER regime.

The paper is organized as follows. Section III presents a detailed setup of different HARQ approaches and provides the assumptions for the link-level simulations. Furthermore, specific optimizations for different HARQ approaches are presented. In Section III-A the evaluation methodology for the purpose of performance comparison is explained. In Section [II] the results of the simulations are presented. In the first part, proactive HARQ with and without prediction is compared
TABLE I

LINK-LEVEL SIMULATION ASSUMPTIONS FOR TRAINING AND TEST SET GENERATION

\begin{tabular}{l|l} 
Number of TBs & $1.7 \mathrm{M}(0.85 \mathrm{M}$ train, $0.85 \mathrm{M}$ test $)$ \\
\hline TB size in bits $\left(N_{\mathrm{Bits}}\right)$ & $360,500,800,1000$ \\
\hline Transmission duration RV $\left(\delta_{\mathrm{t}}\right)$ & 1 OFDM symbol \\
\hline Delay budgets $\left(\delta_{\mathrm{bud}}\right)$ & $14 \delta_{\mathrm{t}}$, i.e. $1 \mathrm{~ms}$, \\
& $28 \delta_{\mathrm{t}}$, i.e. $2 \mathrm{~ms}$ \\
\hline Feedback delays $\left(\delta_{\mathrm{fb}}\right)$ & $2-5$ for $\delta_{\mathrm{bud}}=14$, \\
& $5-12$ for $\delta_{\text {bud }}=28$ \\
\hline Target BLERs $\left(\epsilon_{\mathrm{target}}\right)$ & $10^{-1}, 5 \cdot 10^{-2}, 10^{-2}\left(\delta_{\mathrm{bud}}=14\right)$ \\
& $10^{-1}, 10^{-2}, 10^{-3}\left(\delta_{\mathrm{bud}}=28\right)$ \\
\hline Transmission bandwidth & $1.08 \mathrm{MHz}(6 \mathrm{RBs})$ \\
\hline Channel Code & Rate-1/5 LDPC $(\mathrm{see}[20])$ \\
\hline Modulation order and algorithm & $4-\mathrm{QAM}, 16-\mathrm{QAM}, 64-\mathrm{QAM}$, \\
& Approximated LLR \\
\hline Power allocation & Constant $E_{b} / N_{0}$ \\
\hline Waveform & $3 \mathrm{GPP}$ OFDM, \\
& normal cyclic-prefix, \\
& $15 \mathrm{kHz}$ subcarrier spacing \\
\hline Channel type & 1 Tx $1 \mathrm{Rx}$, TDL-C 100 ns, \\
& $2.9 \mathrm{GHz}, 3.0 \mathrm{~km} / \mathrm{h}$ \\
\hline Equalizer & Frequency domain MMSE \\
\hline Decoder type & Min-Sum $(50$ iterations $)$ \\
\hline Prediction iterations & 5
\end{tabular}

under the assumption that the feedback delay parameter is fixed. In the second part, the performance of proactive HARQ is analyzed with and without prediction, assuming a shortened feedback delay for the prediction-based scheme, whereas in the last part proactive HARQ is compared to reactive HARQ.

\section{SySTEM MODEL}

Table I summarizes the link-level parameters which have been used for the simulations. We analyzed two different latency constraints, a short delay budget of one slot, i.e. 14 OFDM symbols, and a long delay budget of two slots, i.e. 28 OFDM symbols. However, these delay budgets can be arbitrarily scaled. Under these constraints, we evaluated three different HARQ approaches, i.e. proactive HARQ (paHARQ), proactive HARQ with prediction (prHARQ) and reactive HARQ (reHARQ) for multiple TB sizes, a variety of target BLER $\epsilon_{\text {target }}$, and different assumptions on the feedback delay $\delta_{\mathrm{fb}}$. The Signal-to-Noise Ratios (SNRs) for the specific scenarios have been chosen such that the target BLER is achievable. It is important to note that the feedback delay is comprised of multiple delay components, such as the propagation delay, the processing delay, i.e. decoding, and the feedback delay. The prediction is able to reduce the processing delay, by performing only 5 instead of 50 decoding iterations [17]. To maintain the full picture of the proposed approach, we evaluated the performance of the predictionbased scheme under two scenarios: with typical processing delay and a shortened one. In Section $\amalg I-A$ we assumed that the feedback delay stays the same, designated as prHARQ, to show that there is an advantage for a certain parameter range even under this setup. In Section [II-B, we compared the performance of the prediction-based approach assuming that the shorter processing delay reduces the feedback delay 


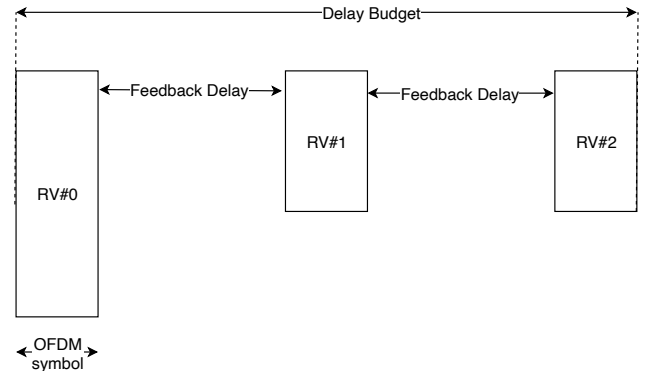

Fig. 1. Schematic representation of reHARQ without any power or bandwidth constraint.

by one OFDM symbol, designated as early proactive HARQ with prediction (eprHARQ).

\section{A. Evaluation Methodology}

In addition to achieving the reliability and the latency targets which are mandatory requirements, the performance of the HARQ schemes can be compared in terms of energy efficiency, which is critical for battery powered devices. Based on the renewal-reward theorem [21], the energy efficiency as the number of bits that can be transmitted with a given amount of energy is expressed as:

$$
\eta=\frac{\mathbb{E}[\mathcal{R}]}{\mathbb{E}[\psi]}
$$

where $\mathbb{E}[\mathcal{R}]$ is an expected reward and $\mathbb{E}[\psi]$ is an expected consumed energy, where $\mathbb{E}[\psi]:=\mathbb{E}\left[T_{\mathrm{HARQ}} \cdot P_{\mathrm{RV}}\right]$ with $T_{\mathrm{HARQ}}$ is a number of required transmissions, as defined for the different HARQ schemes in the further in the subsections, and $P_{\mathrm{RV}}$ is a consumed energy for an RV which is constant for the proactive HARQ schemes. Furthermore, the reward is $\mathcal{R}:=0$ in case the transmission failed within the latency budget and $\mathcal{R}:=N_{\text {Bits }}$ in case the transmission was successful. Hence, the expected reward is given as $\mathbb{E}[\mathcal{R}]:=(1-\epsilon) N_{\text {Bits }}$, where $\epsilon$ is an associated total error probability.

In order to compare different HARQ schemes, we evaluated the Energy Efficiency Gain (EEG) defined as:

$$
\Theta_{\mathrm{H} 1, \mathrm{H} 2}:=\frac{\eta_{\mathrm{H} 1}-\eta_{\mathrm{H} 2}}{\eta_{\mathrm{H} 1}},
$$

where $\eta_{\mathrm{H} 1}$ and $\eta_{\mathrm{H} 2}$ are the expected energy efficiency values of the two selected HARQ approaches.

\section{B. Reactive $H A R Q$}

The reHARQ approach is based on IR HARQ [7], and as was mentioned before is not able to achieve the required BLER target within the given power limitation at the evaluated SNR values. However, since it is a widely used state-of-the-art mechanism in 5G, a comparison is justified although the constraints are not the same. As depicted in Fig. 11, each of the data re-/transmissions can be reallocated to a transmission, which is as short as a single OFDM symbol, to reduce the delay as much as possible. Furthermore, the distribution of redundancy over the different re-/transmissions can be optimized such that the expected number of overall transmissions is minimized.

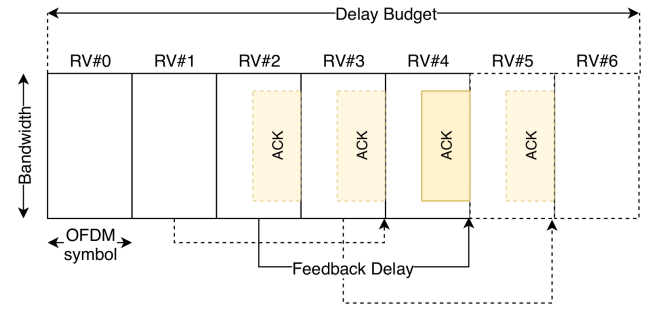

Fig. 2. Schematic representation of paHARQ with bandwidth limitation.

The expected number of transmissions, where a transmission is equivalent to an $\mathrm{RV}$ for the proactive approaches to ensure comparability, is defined as:

$$
\mathbb{E}\left[T_{\mathrm{reHARQ}}\right]=\sum_{i=0}^{n_{\max }} \epsilon_{i} T_{i},
$$

where $n_{\max }$ is a maximum number of HARQ re-transmissions within the latency budget and $\epsilon_{i}$, and $T_{i}$ are an associated error probability of a single transmission and a number of paHARQequivalent RVs, respectively.

\section{Proactive HARQ}

In Rel. 16 URLLC, 3GPP adopted different HARQ approaches including proactive HARQ to enable HARQ for Configured Grants (CGs) in latency constrained scenarios [7]. The proactive HARQ approach first determines a number of RVs to achieve the target error rate. As depicted in Fig. 2, these RVs are transmitted consecutively up to a reception of an ACK which terminates the transmission process. This approach ensures the reception of the packet within the delay budget. However, the efficiency is determined by the feedback delay, also designated as the HARQ RTT. Since the receiver has to process the received signal stream to generate the feedback, at the time moment when the ACK message reaches the transmitter, multiple RVs have already been sent. Thus, despite overcoming the HARQ-specific issue of the feedback delay in latency constrained scenarios, the gained latency comes from trading off the spectral efficiency due to unnecessary retransmissions. Hence, the expected number of transmissions is designated as:

$$
\mathbb{E}\left[T_{\text {paHARQ }}\right]=\sum_{i=1}^{n-\delta_{\mathrm{fb}}} P_{i}\left(i+\delta_{\mathrm{fb}}\right),
$$

where $n$ is a maximum number of transmissions, $\delta_{\mathrm{fb}}$ is the feedback delay, and $P_{i}:=\left(\prod_{k=1}^{i-1} \epsilon_{k}\right)\left(1-\epsilon_{i}\right)$ is the probability that the packet is decodable at the $i$-th RV with $\epsilon_{n}:=0$.

\section{Novel Proactive HARQ with Prediction}

The proactive HARQ with prediction uses the same setup, as depicted in Fig. 2 The major difference is that the full decoding of an RV is replaced firstly by a small number of decoding iterations on the received subcode, and secondly, a logistic regression predictor that uses Variable Node Reliabilities (VNRs) as an input vector [17], [22]. Previous work has 


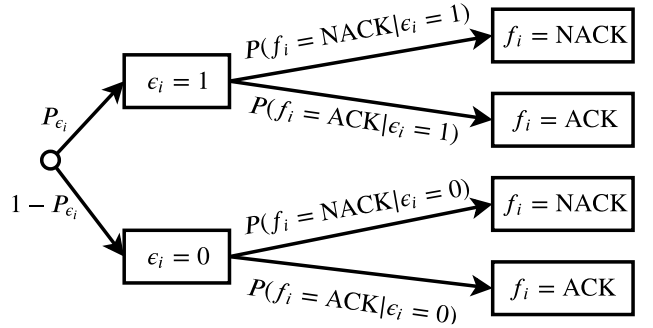

Fig. 3. Probabilistic model of HARQ feedback prediction.

showed that a logistic regression achieves the best performance among a variety of predictors [17]. Due to the paper's length limitation we leave the basic definitions, such as subcode, false negative/positive probabilities, out of scope of this paper. Please refer to [22] for more details.

Fig. 3 shows a probabilistic model, which has been used to evaluate the performance of the prHARQ approach. In this model the error probabilities have been obtained by MonteCarlo link-level simulations, and the false-positive and falsenegative prediction probabilities were found by firstly training the logistic regression on half of the simulation data and then deriving them based on the remaining data set.

1) Parameter selection for Predictive IR HARQ: False predictions, false-positive as well as false-negative, impact the performance of the prHARQ scheme. False-positive mispredictions increase the total BLER, whereas false-negative mispredictions mainly cause unnecessary retransmissions, and hence degrade the energy efficiency. In order to optimize the HARQ operation the following optimization problem has to be solved:

$$
\begin{array}{ll}
\underset{P_{\mathrm{fp}}^{n}}{\operatorname{minimize}} & \mathbb{E}\left[T_{\mathrm{prHARQ}}\left(P_{\mathrm{fp}}^{n}\right)\right] \\
\text { subject to } & \epsilon\left(P_{\mathrm{fp}}^{n}\right) \leq \epsilon_{\text {target }},
\end{array}
$$

where $P_{\mathrm{fp}}^{n}:=\left(P_{\mathrm{fp}_{1}}, \ldots, P_{\mathrm{fp}_{i}}, \ldots, P_{\mathrm{fp}_{n}}\right)$ with $P_{\mathrm{fp}_{i}}:=P\left(f_{i}=\right.$ $\left.\mathrm{ACK} \mid \epsilon_{i}=1\right)$ are the false-positive prediction error probabilities, which are used to compute the false-negative prediction error probabilities: $P_{\mathrm{fn}_{i}}:=P\left(f_{i}=\mathrm{NACK} \mid \epsilon_{i}=0\right)=f\left(P_{\mathrm{fp}_{i}}\right)$. Furthermore, $T\left(P_{\mathrm{fp}}^{n}\right) \in\left\{T_{\min }+\delta_{\mathrm{fb}}, T_{\min }+\delta_{\mathrm{fb}}+1, \ldots, T_{\max }\right\}$ is a random variable representing the number of required transmissions. For the purpose of simplification, let $K \in$ $\left\{0, \ldots, k_{\max }\right\}$ be a dependent random variable with $T\left(P_{\mathrm{fp}}^{n}\right)=$ $T_{\min }+K+\delta_{\mathrm{fb}}$ and $k_{\max }:=T_{\max }-T_{\min }-\delta_{\mathrm{fb}}$. Hence, the probability distribution of $T\left(P_{\mathrm{fp}}^{n}\right)$ is given as follows:

$$
\mathbb{P}\left[T\left(P_{\mathrm{fp}}^{n}\right)=t\right]:=\mathbb{P}[K=k]=P_{1}(k),
$$

where $P_{i}(k)$ with $i \in\left\{1, \ldots, k_{\max }+1\right\}$ is $P_{k+1}(k):=1$ for $i=k+1$ and $k=k_{\max }$, and otherwise is defined recursively as:

$P_{i}(k):= \begin{cases}P_{\epsilon_{i}}\left(1-P_{\mathrm{fp}_{i}}\right) P_{i+1}, & \text { if } i<k, \\ P_{\epsilon_{i}}\left(1-P_{\mathrm{fp}_{i}}\right) P_{i+1}+\left(1-P_{\epsilon_{i}}\right) P_{\mathrm{fn}_{i}}, & \text { if } i=k, \\ P_{\epsilon_{i}}\left(1-P_{\mathrm{fp}_{i}}\right)+\left(1-P_{\epsilon_{i}}\right) P_{\mathrm{fn}_{i}}, & \text { if } i=k+1 .\end{cases}$

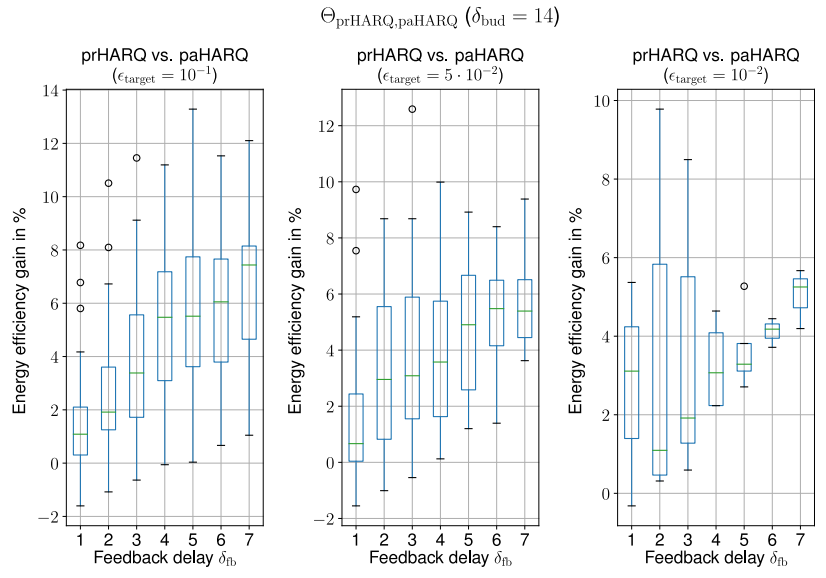

Fig. 4. EEG of the prHARQ approach compared to the paHARQ over different feedback lengths for all transport block sizes, SNRs, and $\delta_{\text {bud }}=14$.

Furthermore, the total BLER $\epsilon\left(P_{\mathrm{fp}}^{n}\right)$ is defined as follows:

$$
\epsilon\left(P_{\mathrm{fp}}^{n}\right):=\left(\prod_{i=1}^{\delta_{\mathrm{fb}}} P_{\epsilon_{i}}\right) P_{\mathrm{e}}\left(\delta_{\mathrm{fb}}+1\right),
$$

with $P_{\mathrm{e}}(i)$ as an error probability at a certain $\mathrm{RV}$, which is $P_{\mathrm{e}}\left(T_{\max }\right):=P_{\epsilon_{i}}$ for $i=T_{\max }$, and otherwise is defined recursively as:

$$
P_{\mathrm{e}}(i):=P_{\epsilon_{i}}\left(P_{\mathrm{fp}_{i}}+\left(1-P_{\mathrm{fp}_{i}}\right) P_{\mathrm{e}}(i+1)\right) .
$$

The stated problem in 5 is a multi-variate optimization problem with non-linear constraints. Since the functional mapping between the false-positive and the false-negative error probabilities is unknown, however can be extracted numerically from the link-level simulations, we used the trustregion constrained algorithm to find local minima to the stated problem with non-linear constraints [23]. A random starting point has been chosen and the Monte-Carlo method has been used to find a near-optimal solution.

\section{RESULTS}

We performed link-level simulations to evaluate the efficiency of the different HARQ schemes. A variety of scenarios with different SNRs, target BLERs, TB sizes and feedback delays have been modelled and the EEGs of the different HARQ schemes have been determined individually for each scenario. In the first part, we evaluated the HARQ approaches under the assumption of the same feedback delay for all HARQ schemes. In the second part, we compared the performance of the prHARQ scheme to the paHARQ, assuming a feedback delay reduction by one time unit $\delta_{\mathrm{t}}$, i.e. one OFDM symbol.

\section{A. Performance evaluation of the HARQ schemes with same feedback delay}

Fig. 4 presents the EEG of prHARQ compared to paHARQ over different feedback delays for three target BLERs. The EEG stays in approximately the same range for all three BLER targets. It is clearly visible that the average EEG shows a gain for all feedback delays. For feedback delays $\delta_{\mathrm{fb}}$ larger than 

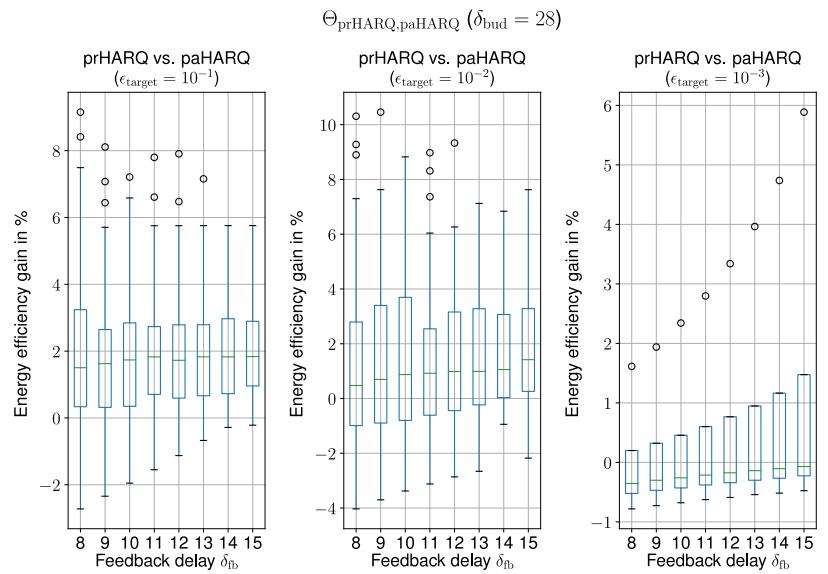

Fig. 5. EEG of the prHARQ approach compared to the paHARQ over different feedback lengths for all transport block sizes, SNRs, and $\delta_{\text {bud }}=28$.

three, the EEG is solely positive for even all evaluation points. Furthermore, an increase of the gain for higher feedback delays is consistently notable for all the target error rates. Hence, it can be concluded that the prediction provides a significant efficiency gain compared to the non-predictive case even under the assumption of no processing time reduction for the prediction.

As can be seen in Fig. 5, the EEG is in a similar, however slightly smaller, range for the delay budget of 28 OFDM symbols for the different BLER targets as compared to the more stringent latency budget. For the target error rate of $\epsilon_{\text {target }}=10^{-1}$, the EEG is mainly in the range of $0 \%-3 \%$ with a slightly increasing trend for higher feedback delays. The EEG increases on average for higher feedback delays. For the lowest target error rate of $\epsilon_{\text {target }}=10^{-3}$, the EEG decreases generally compared to the higher BLER regimes. In contrast to the scenario of a very stringent latency target, there are more evaluation points with a negative gain than a positive one even for high feedback delays. This suggests that feedback prediction without processing time reduction compared to full decoding may only be beneficial under certain circumstances, such as a very tight latency target and rather higher BLERs. In Fig. 6, the same trends are also observable for the mean EEG. While showing solely a positive gain for both latency constraints, the mean EEG of the very stringent delay budget is clearly higher compared to less stringent one. In the very stringent scenario, the prHARQ approach performs better at lower BLER targets for very small feedback delays whereas this trend changes to the contrary at medium and high feedback delays which hints to that the prediction accuracy is decreasing relatively stronger with increasing feedback delay in the lower BLER regime.

\section{B. Evaluation of early feedback}

One of the major motivations to perform a low-complexity prediction is to reduce the processing time and, thus reduce the feedback delay. In this section, we analyze the gain of the eprHARQ scheme assuming a feedback delay reduced by one

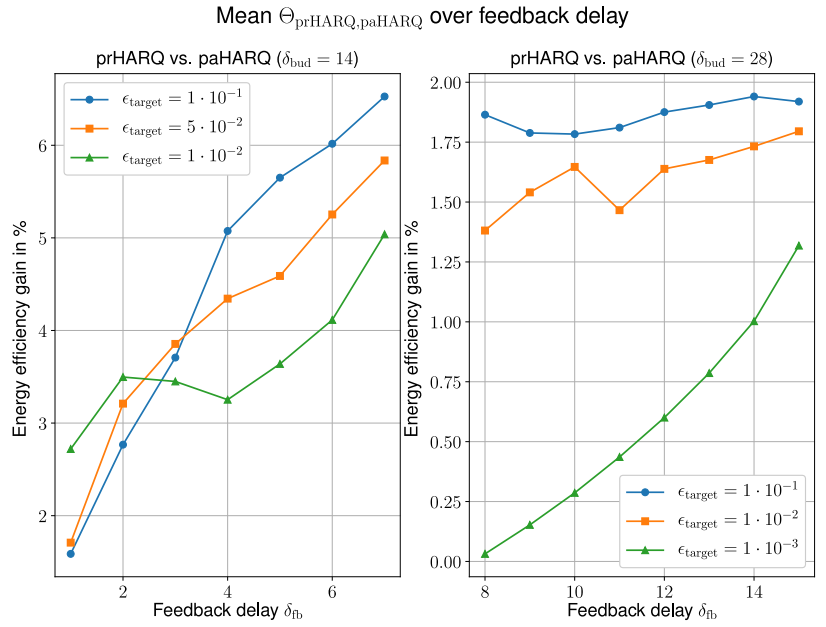

Fig. 6. Mean EEG of the prHARQ approach compared to the paHARQ over different feedback lengths.
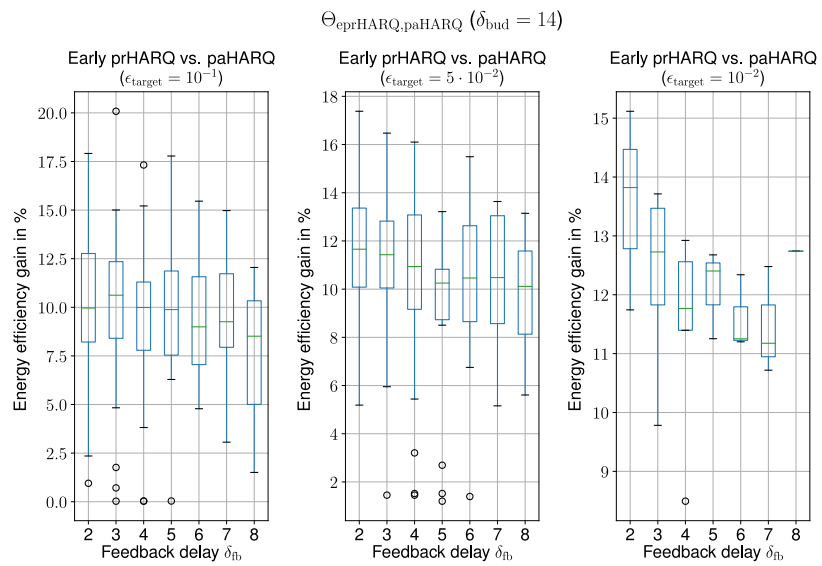

Fig. 7. EEG of the eprHARQ approach compared to the paHARQ over different feedback lengths for all transport block sizes, SNRs, and $\delta_{\text {bud }}=14$.

time unit, i.e. one OFDM symbol. In Fig. 7 the EEG is shown over the feedback delay of the paHARQ scheme for $\delta_{\text {bud }}=14$. As clearly notable, the eprHARQ scheme profits significantly from the reduced feedback delay and the EEG is for most of the evaluation points in the range of approximately $8 \%$ and $14 \%$. In general, the lower target BLERs profit even more from the reduced feedback delay. However in contrast to the case without processing time reduction, higher feedback delays profit less than lower feedback delays. This is explainable by the fact that the reduction of the feedback delay by one OFDM symbol is relatively smaller for higher feedback delays. Overall, a clear benefit for the energy efficiency of the eprHARQ scheme can be observed.

The same effects are also observable for the latency budget of 28 OFDM symbols in Fig. 8 However, compared to the more stringent latency constraint the eprHARQ profits less overall. This can be explained by the relatively smaller reduction of the feedback delay compared to the latency budget. Nevertheless, the EEG is in the range of approximately $1 \%$ up to $12 \%$ for most evaluation points. For the lowest target BLER 

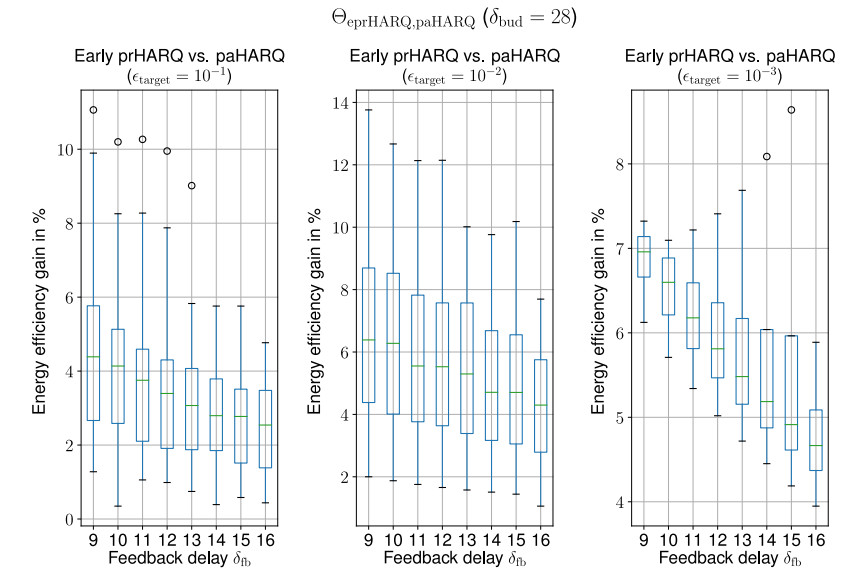

Fig. 8. EEG of the eprHARQ approach compared to the paHARQ over different feedback lengths for all transport block sizes, SNRs, and $\delta_{\text {bud }}=28$.
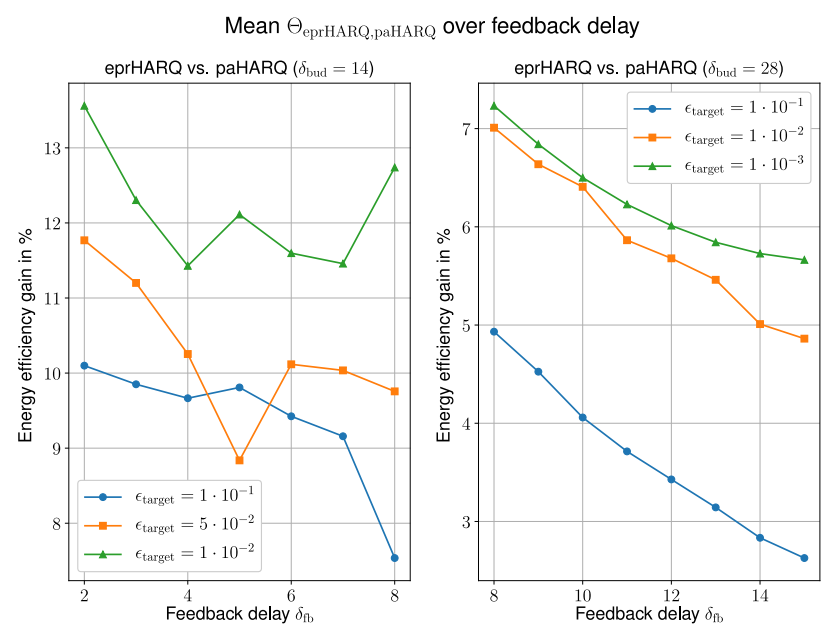

Fig. 9. Mean EEG of the eprHARQ approach compared to the paHARQ over different feedback lengths.

of $\epsilon_{\text {target }}=10^{-3}$, a significant EEG between $4.5 \%$ up to $7.5 \%$ is achieved by the eprHARQ scheme, where the gain tends to decrease with an increasing feedback delay. To summarize eprHARQ achieves a significant EEG being slightly larger for the very stringent latency requirement compared to the less stringent one. This suggests that the strength of eprHARQ mainly lies in scenarios with low BLER targets and very stringent latency requirements making it a perfect candidate technique for IIOT scenarios. Fig. 9 shows the mean EEG over the different feedback delays. As already noted in the previous figures, the mean EEG is decreasing with increasing feedback delay which hints to that the processing time reduction becomes relatively less significant with increasing feedback delay. However, the eprHARQ approach achieves a significant mean EEG for both latency constraints over all BLER targets and all feedback delays, especially in the lower BLER regime as noted previously.

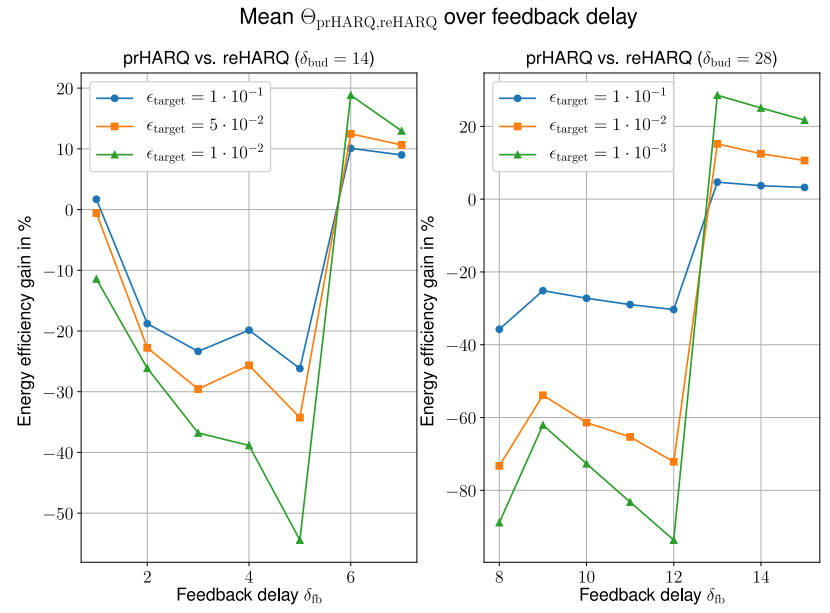

Fig. 10. Mean EEG of the prHARQ approach compared to the reHARQ without power constraint over different feedback lengths for all transport block sizes, SNRs, and delay budgets.

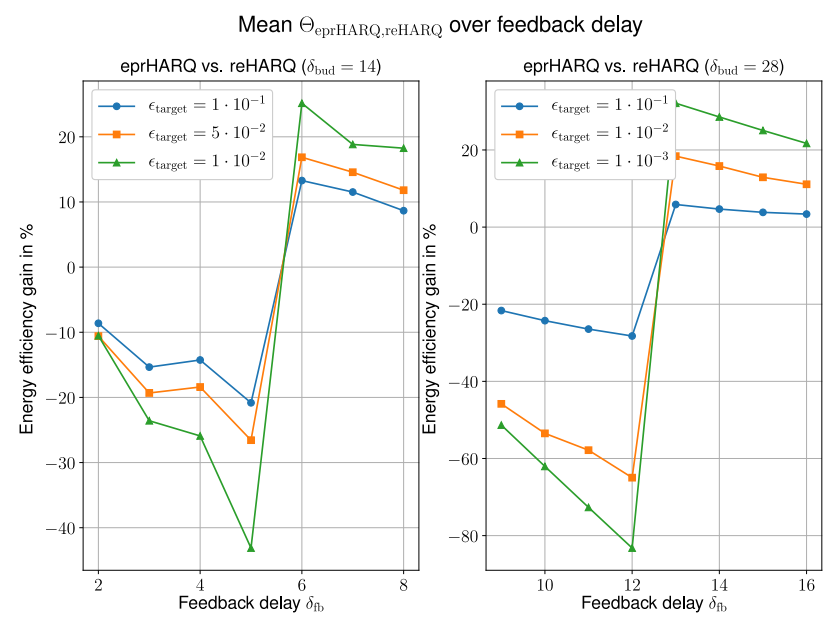

Fig. 11. Mean EEG of the eprHARQ approach compared to the reHARQ without power constraint over different feedback lengths for all transport block sizes, SNRs, and delay budgets.

\section{Comparison of proactive and early proactive $H A R Q$ with reactive $H A R Q$}

In Fig. 10 the mean EEG of prHARQ compared to reHARQ is shown over the feedback delay. As explained previously, the performance of the reHARQ scheme is obtained under the assumption of no power limitations at the transmitter device. Still a clear positive gain of the prHARQ approach is notable for sufficiently high feedback delays of 6 and 13 for a latency budget of 14 and 28, respectively. A higher gain for lower target BLERs is clearly notable, which is due to the low efficiency of the reHARQ in this particular case. For smaller feedback delays, reHARQ clearly outperforms the prHARQ scheme by avoiding unnecessary retransmissions. However, due to power or complexity limitations the reHARQ scheme may not be suitable for IIOT devices, as discussed above. In Fig. 11, we can see for the early feedback case that the eprHARQ outperforms the reHARQ again at the feedback 
delays of 6 and 13 for more stringent and less stringent delay budget, respectively. Compared to the proactive scheme without early feedback, it is observable that the efficiency gain increases at the same feedback delays, which is due to the early prediction. Despite to the higher complexity and power requirements, reHARQ is outperformed by proactive HARQ with or without processing time reduction at sufficiently large feedback delays.

\section{CONCLUSION}

We evaluated the energy efficiency of prHARQ compared to paHARQ. We showed that the prediction improves the energy consumption, demonstrating more advantage for a higher BLER and less advantage for a lower BLER at least if no processing time reduction is considered. In general, the performance of the prHARQ improved for increasing feedback delays hinting at the benefits of the prediction to cope especially with high feedback delays. In the second part, we showed that considering a small feedback delay reduction by one OFDM symbol for the feedback prediction mechanism, the EEG increases significantly in favor of the eprHARQ. Especially in the low BLER regime, the energy efficiency is increased by $11 \%$ up to $15 \%$ and $4 \%$ up to $7.5 \%$ for the very stringent and stringent latency constraint, respectively. Hence, also the energy consumption is reduced significantly. In the last part, we demonstrated that even unconstrained reHARQ cannot outperform power-constrained prHARQ for sufficiently large feedback delays of 6 and 13 OFDM symbols for the more stringent and the less stringent latency budget, respectively.

\section{REFERENCES}

[1] N. S. B. Nokia, "Enhanced Industrial Internet of Things (IoT) and URLLC support," 3GPP, Tech. Rep. RP-193233, Dec. 2019.

[2] Ericsson, "New SID on Support of Reduced Capability NR Devices," 3GPP, Tech. Rep. RP-193238, Dec. 2019.

[3] B. Bertenyi, S. Nagata, H. Kooropaty, X. Zhou, W. Chen, Y. Kim, $\mathrm{X}$. Dai, and $\mathrm{X}$. Xu, "5g nr radio interface," Journal of ICT Standardization, vol. 6, no. 3, pp. 31-58, May 2018. [Online]. Available: https://doi.org/10.13052/jicts2245-800X.613

[4] 3rd Generation Partnership Project, "Study on physical layer enhancements for NR ultra-reliable and low latency case (URLLC)," 3GPP, Tech. Rep. TS38.824, Mar. 2019.

[5] P. Wu and N. Jindal, "Performance of hybrid-arq in block-fading channels: A fixed outage probability analysis," IEEE Transactions on Communications, vol. 58, no. 4, pp. 1129-1141, 2010.

[6] L. Vangelista and M. Centenaro, "Performance evaluation of harq schemes for the internet of things," Computers, vol. 7, no. 4, 2018. [Online]. Available: https://www.mdpi.com/2073-431X/7/4/48

[7] N. H. Mahmood, R. Abreu, R. Böhnke, M. Schubert, G. Berardinelli, and T. H. Jacobsen, "Uplink grant-free access solutions for urllc services in 5g new radio," in 2019 16th International Symposium on Wireless Communication Systems (ISWCS), Aug. 2019, pp. 607-612.

[8] T. Jacobsen, R. Abreu, G. Berardinelli, K. Pedersen, P. Mogensen, I. Z. Kovacs, and T. K. Madsen, "System level analysis of uplink grantfree transmission for urllc," in 2017 IEEE Globecom Workshops (GC Wkshps), Dec. 2017, pp. 1-6.

[9] Y. Liu, Y. Deng, M. Elkashlan, A. Nallanathan, and G. Karagiannidis, "Analyzing grant-free access for urllc service," IEEE Journal on Selected Areas in Communications, vol. PP, Aug. 2020.

[10] B. Makki, T. Svensson, G. Caire, and M. Zorzi, "Fast harq over finite blocklength codes: A technique for low-latency reliable communication," IEEE Transactions on Wireless Communications, vol. 18, no. 1, pp. 194209, 2019
[11] Z. Hou, C. She, Y. Li, L. Zhuo, and B. Vucetic, "Prediction and communication co-design for ultra-reliable and low-latency communications," IEEE Transactions on Wireless Communications, vol. 19, no. 2, pp. 1196-1209, 2020.

[12] T. V. K. Chaitanya, "Harq systems: Resource allocation, feedback error protection, and bits-to-symbol mappings," Ph.D. dissertation, Linköping University Electronic Presss, Linköping, 2013.

[13] J. Nadas, P. Klaine, L. Zhang, G. Zhao, M. Imran, and R. Souza, "Performance analysis of early-harq for finite block-length packet transmission," in 2019 IEEE International Conference on Industrial Cyber Physical Systems (ICPS), 2019, pp. 391-396.

[14] G. Berardinelli, S. R. Khosravirad, K. I. Pedersen, F. Frederiksen, and P. Mogensen, "Enabling Early HARQ Feedback in 5G Networks," in 83rd IEEE Vehicular Technology Conference (VTC Spring), May 2016, pp. 1-5.

[15] G. Berardinelli, S. R. Khosravirad, K. I. Pedersen, F. Frederiksen, and P. Mogensen, "On the benefits of early HARQ feedback with non-ideal prediction in 5G networks," in International Symposium on Wireless Communication Systems (ISWCS), Sep. 2016, pp. 11-15.

[16] S. Sesia, G. Caire, and G. Vivier, "Incremental redundancy hybrid arq schemes based on low-density parity-check codes," IEEE Transactions on Communications, vol. 52, no. 8, pp. 1311-1321, 2004.

[17] N. Strodthoff, B. Göktepe, T. Schierl, C. Hellge, and W. Samek, "Enhanced machine learning techniques for early harq feedback prediction in 5g," IEEE Journal on Selected Areas in Communications, vol. 37, no. 11 , pp. 2573-2587, 2019.

[18] S. Khalili and O. Simeone, "Uplink harq for cloud ran via separation of control and data planes," IEEE Transactions on Vehicular Technology, vol. 66, no. 5, pp. 4005-4016, 2017.

[19] P. Rost and A. Prasad, "Opportunistic hybrid arq-enabler of centralized-ran over nonideal backhaul," IEEE Wireless Communications Letters, vol. 3, no. 5, pp. 481-484, 2014.

[20] MCC Support, “3GPP TS 38.212 v16.0.0," 3GPP, Tech. Rep., Jan. 2020, pp. 19-30.

[21] S. Ross, Stochastic processes, ser. Wiley series in probability and statistics: Probability and statistics. Wiley, 1996.

[22] B. Göktepe, S. Fähse, L. Thiele, T. Schierl, and C. Hellge, "Subcodebased early HARQ for 5G," in IEEE International Conference on Communications (ICC) Workshops, May 2018.

[23] A. R. Conn, N. I. M. Gould, and P. L. Toint, Trust Region Methods. Society for Industrial and Applied Mathematics, 2000. [Online]. Available: https://epubs.siam.org/doi/abs/10.1137/1.9780898719857 